\title{
The Dynamic Role of Breathing and Cellular Membrane Potentials in the Experience of Consciousness
}

\author{
Ravinder Jerath $^{1 *}$, Shannon M. Cearley ${ }^{1}$, Vernon A. Barnes ${ }^{2}$, Santiago Junca ${ }^{3}$ \\ ${ }^{1}$ Charitable Medical Healthcare Foundation, Augusta, GA, USA \\ ${ }^{2}$ Georgia Prevention Institute, Augusta University, Augusta, GA, USA \\ ${ }^{3}$ Santiago Junca, Medical Illustration, Tucker, GA, USA \\ Email: *rj605r@aol.com
}

How to cite this paper: Jerath, R., Cearley, S.M., Barnes, V.A. and Junca, S. (2017) The Dynamic Role of Breathing and Cellular Membrane Potentials in the Experience of Consciousness. World Journal of Neuroscience, 7, 66-81.

https://doi.org/10.4236/wjns.2017.71007

Received: December 8, 2016

Accepted: January 22, 2017

Published: January 25, 2017

Copyright $\odot 2017$ by authors and Scientific Research Publishing Inc. This work is licensed under the Creative Commons Attribution International License (CC BY 4.0).

http://creativecommons.org/licenses/by/4.0/ (c) (i) Open Access

\begin{abstract}
Understanding the mechanics of consciousness remains one of the most important challenges in modern cognitive science. One key step toward understanding consciousness is to associate unconscious physiological processes with subjective experiences of sensory, motor, and emotional contents. This article explores the role of various cellular membrane potential differences and how they give rise to the dynamic infrastructure of conscious experience. This article explains that consciousness is a body-wide, biological process not limited to individual organs because the mind and body are unified as one entity; therefore, no single location of consciousness can be pinpointed. Consciousness exists throughout the entire body, and unified consciousness is experienced and maintained through dynamic repolarization during inhalation and expiration. Extant knowledge is reviewed to provide insight into how differences in cellular membrane potential play a vital role in the triggering of neural and non-neural oscillations. The role of dynamic cellular membrane potentials in the activity of the central nervous system, peripheral nervous system, cardiorespiratory system, and various other tissues (such as muscles and sensory organs) in the physiology of consciousness is also explored. Inspiration and expiration are accompanied by oscillating membrane potentials throughout all cells and play a vital role in subconscious human perception of feelings and states of mind. In addition, the role of the brainstem, hypothalamus, and complete nervous system (central, peripheral, and autonomic) within the mind-body space combine to allow consciousness to emerge and to come alive. This concept departs from the notion that the brain is the only organ that gives rise to consciousness.
\end{abstract}

\section{Keywords}

Membrane Potential, Unified Consciousness, Respiration, Thalamus, Oscillations 


\section{Introduction}

This review article serves to introduce consciousness as a living entity that is maintained through respiration-the sum of the combination of not only the cells of the internal and external body, but also the cellular and biological processes that take place within the brain, eyes, heart, lungs, and intact body. We contend that 1 ) every mechanism within the body works in unison to bring alive the tripartite complexity of the mind, brain, and body through sensory organs, cardiorespiratory synchronization, and oscillatory activity, and 2) these activities collectively and seamlessly aggregate to create a homogenous consciousness experience. Here, we propose that consciousness is not located in or around a single organ, but rather permeates every cell of the body in reciprocal communication with the brain and central nervous system. The interconnected and interdependent relationship between the brain and body [1] as we will explain, happens in part through corticothalamic processes and varying polarity of cellular membrane potentials (see Table 1). Membrane potentials are one of the "defining characteristics of living cells", and changes in voltage regulate transduction

Table 1. Varying resting membrane potentials.

\begin{tabular}{|c|c|}
\hline Cell & Resting Membrane Potential \\
\hline Astrocytes & $20 \mathrm{mV}$ higher than surrounding neurons [6] \\
\hline Large peripheral nerve fibers & $-90 \mathrm{mV}[7]$ \\
\hline Ventricular myocyte & $-90 \mathrm{mV}[8]$ \\
\hline Nerve & $-85 \mathrm{mV}$ to $-90 \mathrm{mV}[7][9]$ \\
\hline Heart & $-85 \mathrm{mV}[9]$ \\
\hline Glial syncytium & $-80 \mathrm{mV}$ to $-90 \mathrm{mV}[6]$ \\
\hline Skeletal Muscle & $-80 \mathrm{mV}$ to $-90 \mathrm{mV}$ [7] \\
\hline Spinal motor neuron & $-65 \mathrm{mV}[7]$ \\
\hline All of soma & $-65 \mathrm{mV}[7]$ \\
\hline Layer 5 pyramidal neuron & $-60 \mathrm{mV}$ to $-80 \mathrm{mV}[10]$ \\
\hline Neutrophils & $-60 \mathrm{mV}[11]$ \\
\hline Intestinal smooth muscle & $-50 \mathrm{mV}$ to $-60 \mathrm{mV}$-average is $-56 \mathrm{mV}$ [7] \\
\hline Olfactory cells (unstimulated) & $-55 \mathrm{mV}[7]$ \\
\hline $\begin{array}{c}\text { Primary Tracheobronchial } \\
\text { Human Epithelia-Basolateral }\end{array}$ & $-34 \mathrm{mV}[11]$ \\
\hline Liver & $-28 \mathrm{mV}$ to $-40 \mathrm{mV}$ [9] \\
\hline $\begin{array}{c}\text { Primary Tracheobronchial } \\
\text { Human Epithelia-Apical }\end{array}$ & $-24 \mathrm{mV}[11]$ \\
\hline Human Erythrocyte & $-8.4 \mathrm{mV}$ to $-4.5 \mathrm{mV}[9][12]$ \\
\hline
\end{tabular}

Cellular resting membrane potential ranges from $-90 \mathrm{mV}$ (large peripheral nerve fibers) to -4.5 $\mathrm{mV}$ (red blood cell) but does not result from unbalanced ionic phase flow. Instead it measures the required work to move ions between phases [9]. There is a lack of evidence to support membrane potential in alveolar epithelial cells due to a lack of adequate culture models and complicated anatomy [11]. 
of signals [2]. We propose that dynamic membrane potentials at the cellular level, along with their modulations by inspiration and expiration, play a fundamental role in the experience of a consciousness that is not only unified [3], but is also perceived as a living phenomenon. Western thought emphasizes that consciousness is localized in the brain; however, Eastern thought suggests that the mind and body are one, as realized, for example via meditation and Samkhya-Yoga through introspection [4]. These practices provide experiences that lead us to suggest that not only consciousness is a body-wide experience, but also the mind and body are a homogenous entity linked by dynamic homeostasis [5].

\section{A Layered Consciousness}

Similar to technology and architecture, consciousness is built upon layers. A computer cannot execute the simplest of commands without bits and bytes; a skyscraper cannot be constructed without a strong foundation and proper support beams; analogously, consciousness cannot arise without the subconscious and unconscious physiology. Damage to these foundational layers can lead to partial or complete dysfunction of the whole [13].

There are three neural and non-neural layers that build upon one another in order for consciousness to emerge. The first layer consists of activity within the default mode network, resting state network, reticular activating system, cardiorespiratory system, and is dominated by slow (alpha) oscillations [6] [13]. This activity not only creates the foundation on which other oscillatory activity is built, it also forms the neural sensory memory space. Damage to this layer can lead to unconsciousness, coma, sleep disorders and autonomic dysfunction [6] [13]. The second layer is composed of limbic activity, emotions, feelings, gut and visceral connections, and includes activity from the limbic and cardiorespiratory systems. Theta and beta oscillations dominate this layer, and damage to this layer can contribute to emotional disorders, amnesia, and panic attacks [6] [13]. The third layer is attributed to higher processing and consciousness, and is also responsible for filling in the sensory memory space [6] [13]. Neural activity in this layer is received from cortical, corticothalamic, and cardiorespiratory areas and the dominating oscillations are alpha and gamma. Contralateral neglect, cortical blindness, memory loss (due to stroke), or sensory dysfunction can be the result if this layer is damaged [6] [13]. Each of these layers builds one upon the other, constructing the internal framework for a unified conscious experience through information integration with the thalamus via corticothalamic feedback loops.

The conscious aspect of human behavior and moods are dominated by the unconscious and subconscious activities. For example, Kay et al. explained that in the rat, theta oscillations are not only referred to as respiratory oscillations, but that they are also "driven by sensory" input [14]. In another study by Zelano and colleagues, it was discovered in humans that nasal breathing modulated cognitive function by granting entrance into limbic areas of the brain, and could 
significantly influence "emotion discrimination and recognition memory" [15]. Furthermore, this study reported that breathing could "influence human cognitive processing", while rhythmic breathing patterns could modulate electrical brain activity patterns that regulate behavior, suggesting that there is a possibility that other processes in the body rhythms (i.e. physiological and autonomic) could "shape neural oscillations to optimize human perception, emotion, and cognition" [15]. The study implied that breathing is not simply a passive action mediated by stimulation or attentiveness, but rather it is a promoter of oscillatory synchrony and could "optimize information processing in the brain areas mediating goal-directed behaviors" [15]. These studies suggest that cognitive function and brain activity are both modulated by respiration, thereby supporting our theory that respiration helps facilitate consciousness.

\section{Oscillatory Activity}

The behavior of oscillations at the cellular and subcellular levels directly result from negative feedback loops along with coupled positive and negative feedback loops, indicating that oscillations are the natural end-products of biochemical interactions within the cells [16]. As with facilitating processes associated with embryogenesis, oscillations during growth and adulthood modulate the function of organs and processes of life. Deviant oscillatory patterns can result in developmental defects and or disease [16].

We offer a new perspective in that: 1) oscillations such as cardiorespiratory and brain rhythms may be involved in energy metabolism to various organs [6], 2) oscillations and brain rhythms create an internal reality that allow one to perceive the external and internal world [6] [17], 3) the synchronization or de-synchronization of cardiorespiratory oscillations with the brain stem allow the emotional brain to determine the meaning of the visceral sensations of the chest and gut [18], and 4) corticothalamic oscillations include feed forward and feedback information from the senses that functionally allow a person the sense of awareness and to experience the external world. All cells of the body are electrically charged and connected via gap junctions to form an intra-personal space that subconsciously proxies for the external space that we have termed the " $3 \mathrm{D}$ default space" [6]. Physiology of vision has been studied extensively, and we have proposed a new theory that highlights the primary role of the retina and lateral inhibition [19]. The synchronous oscillatory activity among the thalamic reticular nucleus, the retina, and various locations in the cortex allows one to see a seamless image that subconsciously proxies for external images. The thalamic reticular nucleus unifies the final focusing of the retinal images [19].

\section{Thalamus}

Information transmitted to the cortex is actively governed by the thalamus and the thalamic reticular nucleus, coupled by feedback from the cortex, thus making the thalamus important in cortical processing of peripheral information. This is because all information must pass through the thalamus [20], and sensory in- 
formation must be filled in prior to conscious awareness [6]. The thalamus relays information to the cortex regarding the environment and internal processes, while the cortex communicates with the thalamus concerning output from multiple processing stages [21].

The thalamic reticular nucleus is a thalamic inhibitory agent, regulates corticothalamic networks excitability, and gives rise to some of the rhythms observed in sleep [22]. Neural responses are influenced by inhibition through interactions with membrane dynamics. For instance, relay cells can fire in rapid burst spikes or in tonic trains [23]. Rapid burst spikes occur during low membrane voltage (sleep), are unable to respond to new excitatory input, but are able to recover from inactivation by strong hyperpolarization, and may play a role in vision because they cause cortical excitement [23]. These spikes can transmit separate information from information encoded in single impulses [23]. Bursting increases with drowsiness or inattentiveness, suggesting that bursting acts like a wake-up call, which is significant for information that is relayed through the thalamus [20]. During waking behavior, thalamic relay cells experience tonic and burst firing; however, tonic firing occurs more frequently in alertness and during membrane depolarization (waking) [20] [23].

Cellular communication is supported by gap junctions, which are capable of generating gamma rhythms in inhibitory neurons because "cells with strong electrical coupling can fire synchronously at low frequencies", and they can assist with neuronal calibration in the visual cortex [24]. During sleep and waking states the membrane potential plays a fundamental role in thalamic neurons. Drowsiness begins at approximately $-70 \mathrm{mV}$ and full-blown slow-wave-sleep is induced at $-90 \mathrm{mV}$ [25], suggesting that the thalamus exhibits a lower membrane potential than does the cortex because of the $\sim 20 \mathrm{mV}$ difference between states of waking and sleeping. The difference in membrane potential leads to a change from alpha, beta, and gamma waves to delta waves during deep sleep when 1) the membrane potential is high [26], or 2) from faster rhythms during a waking state to low-frequency rhythms observed during slow-wave and rapid eye movement sleep [24] [27]; however, the role of various EEG waves is not known. During the waking state, the thalamus communicates with the cortex via reciprocal connections-which could be the basis of cognition, and the thalamus is in a mode of tonic firing, which is suspected to be the vehicle for information transfer from the thalamus to the cortex [28]. During slow-wave sleep the thalamus shifts to a burst mode of firing and is associated with the disconnection between the thalamus and cortex [28]. For further explanation on respiration and membrane potentials, please see our previous article discussing widespread depolarization during expiration as a source of respiratory drive [5].

Corticothalamic neurons in the lateral geniculate nucleus and pulvinar may be influenced by neurons in the thalamic reticular nucleus. Neurons in the thalamic reticular nucleus influence corticothalamic neurons in many ways, including: 1) reduction of spike rates via direct inhibition, 2) increase of responses via disinhibition via dendrodentritic synapses, and 3) switching/changing their firing 
modes resulting in how the cortex receives information [21]. Furthermore, it is possible that thalamic reticular neurons: 1 ) affect synchrony and oscillatory patterns of thalamic neurons, and 2) serve as a pacemaker of corticothalamic oscillations because the thalamic reticular nucleus is specifically positioned to assist in cortical synchronization [21]. Cholinergic input to the thalamus mediates alpha oscillations. Additionally, cholinergic inputs may change the synchrony of neurons in the lateral geniculate nucleus because this area contains different groups of neurons that fire at distinct phases of alpha oscillations [21].

\section{Membrane Potentials and the 3D Default Space}

The thalamus is an essential component in the process of consciousness because this hub receives and processes sensory and periphery information, then reprojects it into an external visual field, giving the impression that images and events are external [17]. However, we contend that this information processing must occur in order for a person to understand what is being experienced. The external world is merely a complex, internal recreation based on many factors including glucose and energy metabolism, neurotransmitters, efferent and afferent signals, memory, and attention processes that work together to form the experience of unified consciousness [6].

The three-dimensional (3D) default space is the intrapersonal space [6], which is brought into existence through the body's cellular unity and electrical communication-facilitated by gap junctions and messenger activity through intercellular fluid [29] [30] [31]. This 3D default space consists of the entire brain, body, communicating cells, and the neural sensory memory space [6]. We suggest that the rhythmic oscillations synchronize with those in the periphery and viscera.

The neural sensory memory space is comprised of oscillations from the reticular activating system, the default mode network, and the resting state networks [13]. When the thalamus fills in this space with visual and non-visual representations, and processed sensory information, it allows consciousness to emerge [6]. The consciousness experience occurs when: 1) sensory information from the body and information from the neural sensory memory space is processed through corticothalamic feedback loops, and 2) the thalamus is able to receive and integrate this information to fill in into the 3D default space [6].

We speculate that the highest membrane potentials exist within the astrocytes and neurons located in the brain, and they send electrical energy over long distances (interneurons of the skin and muscles) where the membrane potentials are lower. The oscillations are generated by areas of higher membrane potential that move to areas of lower membrane potential, repeating the process over and over. Once the areas of lower membrane potential increase, the electrical energy moves to the brain where depolarization has occurred as the result of oscillations. This cycle occurs at all levels between the thalamus and cortex, as well as between the thalamus and peripheral neurons. The thalamus is able to communicate with all sensory organs, and perform memory and executive functions 
within 150 milliseconds [32]. We contend this occurs through a flow of back and forth parallel information processing, allowing a person to become aware of his/ her internal and external surroundings while awake.

\section{ATP Formation and Respiration}

Cellular respiration in the mitochondria begins with glycolysis-the process in which glucose is broken down into pyruvic acid, forming adenosine triphosphate (ATP) required for energy [7]. The second stage of glycolysis is the citric acid cycle, and the third stage is the electron transport chain (ETC) [7]. The electrons are stored in the chain and allow for the regulation of potassium channels in both the mitochondria and cell membranes [33]. This membrane potential drives millions of cellular functions, suggesting that electrons are the currency used for cellular and organism survival, because mitochondrial potassium channels regulate mitochondrial respiration and alteration of membrane potential [33].

Energy is essential for: 1) motility, 2) sustaining life, and 3) establishing and maintaining a difference in electrochemical potential between the inside and outside of a cellular membrane-which allows the organism to receive or provide metabolites [34]. Glucose and oxygen create the electrons needed for the electrical potential through glycolysis within the mitochondria (where energy is derived) - and we suggest that all cells require electrical potential for survival. Energy metabolism could be affected by the interaction of slow synchronous oscillations, and cellular and molecular interactions within the body provide energy to neurons and astrocytes [6]. This creates a dynamic infrastructure that allows peripheral areas to transmit information to the thalamus, and to be processed through corticothalamic feedback loops within milliseconds [6]. Thus membrane potential occurs as a result of membrane bound respiratory complexes [34].

Previously it had been proposed that with inspiration the membrane potential of all cells increases, while during expiration it decreases [3]. The metabolic evidence of this correlates with ATP formation during oxygenation and decreases when oxygen availability is lower [35]. We adduce that during the formation of electrons in the ETC, tiny electrons are released, travel via gap junctions, and are directly transmitted to various cells, and thus each breath dynamically raises the electrical potential in the cells maintaining consciousness.

Autonomic (metabolic) breathing (medulla oblongata and pons) is vital to all cellular functions by providing oxygen and energy to all the cells, and autonomic breathing directly corresponds with changes in emotions because of the complex interaction between the brain stem and the limbic and cortical structures [36]. Behavioral breathing has a direct effect on feelings, sense of self, and the emotions through synchronization between breathing and the emotion a person is feeling, because each emotion is associated with a specific breathing pattern [36] [37]. 


\section{Respiration and Membrane Potentials}

Cardiorespiratory synchronization affects the autonomic nervous system and brain activity by increasing homeostatic membrane potential and decreasing intrinsic cellular excitability [5]. This demonstrates that 1) respiration influences brain activity, autonomic nervous system function, blood pressure, and heart activity, and 2) respiration governs homeostatic physiology [3]. This suggests that respiratory neurons in the brainstem are vital components in respiratory control because they generate breathing activity and participate in sensory responses such as fluctuations in oxygen and carbon dioxide.

Respiration consists of an intricate web of intrinsic, synaptic, and modulatory properties that allow the respiratory network to continuously adapt to external and internal environmental changes [38]. Breathing can modulate behavior and behavior can modulate breathing [5] [38] [39]. We propose that breathing is a dynamic and global activity that releases and sends electrical impulses throughout the body (via slowly adapting stretch receptors), and due to the small size of these electrons, they are able to penetrate all of the body's tissues (see Figure 1).

For example, breathing can modulate fear, arousal, and cognitive states; and vocalization, sleep, arousal, fear, exercise, hypoxia, or hypercapnia can modulate breathing [5] [38] [39] [40]. Respiration affects sympathetic and parasympathetic activity by decreasing or increasing cardiorespiratory coherence [3] [41] [42]; therefore, we propose that respiration allows for the constant, real-time communication between the brain and sensory organs.

Inhibition in the cortex, thalamus, hypothalamus, amygdala and reticular activating system takes place during hyperpolarization and includes slow wave sleep [41]. During sleep, the membrane potential of the thalamus increases by $\sim 20 \mathrm{mV}$, severing the connection between the thalamus and cortex [43]. The oscillatory change from alpha and gamma to delta shows a direct relationship between consciousness and membrane potentials, and specific oscillatory activity. We contend that membrane potential in the brain is like a switch, and increasing membrane potential-either through natural occurrence or by artificial meansrenders a person unconscious, as seen in sleep.

The role of respiration in how consciousness is maintained refers to consciousness being supported by both conscious and unconscious physiology, along with the layers of consciousness and unconsciousness that are powered by breathing [6] [13]. Our contention is that respiration causes the dynamic fluctuation observed in cells during repolarization and depolarization, and is vital in cellular function and oscillatory activity. Respiration influences the subconscious-where respiratory activity (through respiratory oscillations) is apparent in the limbic system, limbic cortex, hypothalamus, blood vessels and skin, and peripheral areas (heart and lungs) that are involved in subconscious emotion processing, and in consciousness, where respiration is involved in changes in brain waves [13] [42]. Each emotion has a specific respiratory pattern associated with it, and during inspiration, one is able to intuitively assess the feeling experienced at that moment [37]. The default mode network, cortical cells, and tha- 
lamus are all supported by increased membrane potentials resulting from breathing, and in turn, respiration involved in carrying out short- and long-term activity of the cells [13]. Furthermore, inspiration and expiration are associated with fluctuations in membrane potentials changes that underlie the dynamics of rhythmic breathing [3].
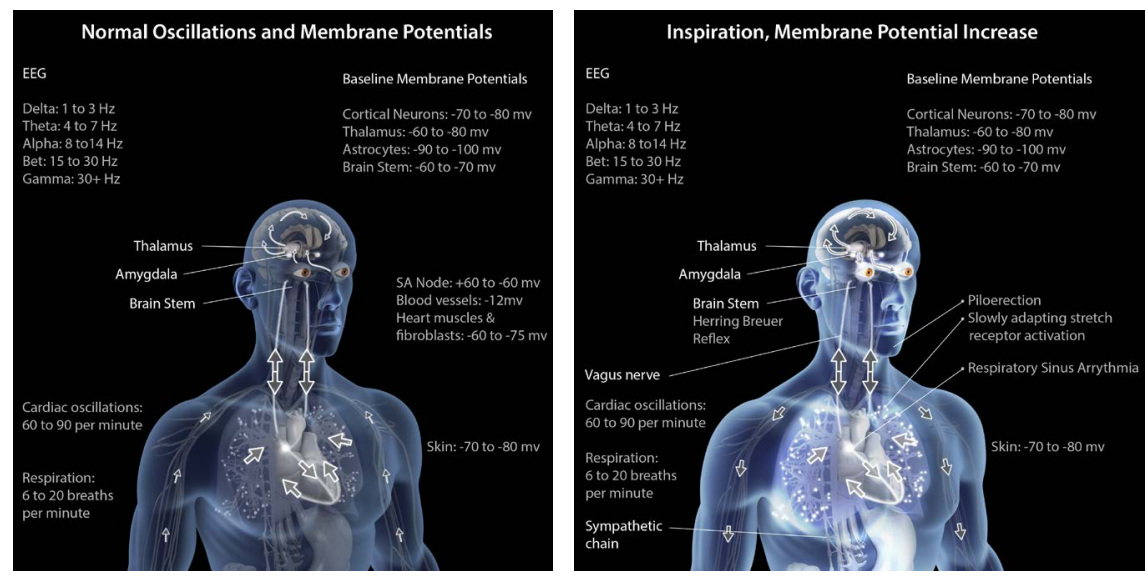

Figure 1 illustrates membrane activity that occurs with inspiration. Breathing is an unconscious activity and normal respiration consists of $6-20$ breaths per minute. During inspiration, an increase in electrical potential causes hyperpolarization in all the body's cells, shown with the white illumination. When inspiration takes place, the intake of oxygen induces cellular respiration which releases electrons from the electron transport chain into the lungs and surrounding tissues, including the heart, blood vessels, nerves, muscles and skin. With inspiration, immediate physiological changes take place: respiratory sinus arrhythmia in the heart, the Herring Breuer Reflex in the brain stem, hyperpolarization and inhibition of sympathetic neurons, activation of slowly adapting stretch receptors in the lungs, and oscillations that lead synchronization with brain waves.

Figure 1. Membrane potential changes during inspiration.

\section{Respiration and Sighing}

Sighing is associated with emotional states [44], such as is observed in states of anxiety where sighing is exaggerated [45]. Furthermore, evidence suggests that excessive sighing can exaggerate panic [45]. Sighing is also considered to be essential for an organism's survival. For example, in experiments using mice that were genetically engineered to lack the ability to sigh, it was found that the mice incapable of sighing died of major lung problems sooner than normal mice [45] [46]. Additionally, research suggests that sighing helps to reset normal breathing patterns, and can "activate the cardiovascular system...by imposing very low frequency oscillations" [44]. These studies support our theory that breathing is vital to cellular functions and oscillatory activity that influence unconscious activities of the heart and lungs that involve emotions. This is because the autonomic nervous system is modulated by increased membrane potential and "decreased intrinsic cellular excitability", and the cardiorespiratory system maintains membrane potentials of neural and non-neural cells throughout the body [5] [6].

\section{Breathing Brings Consciousness to Life}

We contend that the state or quality of awareness would not be possible without 
breathing. The ability to feel at any given moment is possible because the emotional circuits-composed of the limbic system, cardiorespiratory system, and gut - are activated by inspiration and expiration [36]. Cognitive functions performed by the brain such as wakefulness, sense of self, and the executive control system of the mind utilize nearly $20 \%$ of oxygen supplied by a large volume of blood (maintained at approximately $50 \mathrm{~mL} / 100 \mathrm{~g}$ of brain tissue per minute) [47], and $50 \%$ to $80 \%$ of the energy consumed by the brain is utilized in signaling associated with neuronal input and output activity [48].

Unconsciously, breathing activates all cells within the body including internal emotional circuits [36] thereby providing a person with a moment to moment and intuitive assessment of how one "feels". It is well known that during anxiety, breathing rates increase and are shallow, but deep breathing has been shown to help decrease anxiety states [39] [40]. Additionally, the role of rapid breathing is evident in hyperventilation (leading to panic attacks) [49] and breathing into a paper bag has shown to be efficacious in returning respiration to normal [50]. Evidence suggests there is a definitive link between the sympathetic nerve and breathing [51] [52]; therefore, we contend that more research should focus on the link between respiration and sub-threshold oscillations in various organs.

\section{The Role of Meditation in Treating Stress}

Meditation practices including mindfulness-based stress reduction (introduced in 1979) and mindfulness-based cognitive therapy (introduced in 1995), have been shown to use meditation to "effectively manage and treat a range of psychological symptoms (anxiety, depression, emotional responses to stress)" [53]. These types of practices help a person develop the ability to accept his/her experiences instead of finding ways to avoid negative experiences/stimuli [53]. Furthermore, evidence confirms meditation helps to decrease stress levels and attenuate symptoms of: 1) depression, 2) anxiety, 3) negative coping, 4) negative affect, 5) somatization, 6) self-hostility, and 7) post-traumatic stress disorder [53] [54] [55].

Meditation also helps synchronize the heart with respiration. For example, during meditation, the ratio of heartbeats to breaths has been shown to be predominantly 4:1 and 5:1 through slower breathing patterns [56]. This induces a 'relaxation response', termed by Herbert Benson, which can cause instant physiological changes including decreases in: 1) oxygen consumption/carbon dioxide elimination, 2) heart rate, 3) blood pressure, 4) respiratory rate, and 5) sympathetic nervous system reactivity during times of stress [55]. These hemodynamic changes that occur in meditation result from slower breathing patterns and decreased heart rate, which synchronize the heart and lungs, creating a sense calmness [57]. Furthermore, meditation increases neuronal and cellular membrane potentials through cardiorespiratory synchronization, decreasing the intrinsic cellular excitability responsible for activity of the autonomic nervous system and brain [5]. 


\section{Corticomuscular Coherence}

Corticomuscular coherence occurs in all muscles, suggesting a single connection in the complexity of oscillatory interactions in the cortex, thalamus, and cerebellum [58]. Corticomuscular coherence is highly regarded as the method to directly measure the relationship and connection between cortical and muscular activities, and electrophysiological signals are used to measure oscillatory activity within the brain [59]. Corticomuscular oscillations at $\sim 10 \mathrm{~Hz}$ indicate brainmuscle communication, beta band oscillations $(13-30 \mathrm{~Hz})$ are associated with controlling less than maximal muscle force, and low gamma band oscillations $(30-60 \mathrm{~Hz})$ are associated with controlling the production of stronger muscle force [59].

Electroencephalography and magnetoencephalography measure corticomuscular coherence, and the phase locking of oscillations between brain and muscles [60] supports our theory in that peripheral activity is unified with central activity in real-time. This is because synchronous oscillatory activity of alpha, beta, and gamma activity is fundamental for motor control [61]. For example, in experiments using healthy subjects performing isometric muscle contraction tasks, transcranial alternating current stimulation revealed that alpha and low gamma band activity 1) affected one other, and 2) modulated motor cortex and muscle interactions when working together [61].

It is known that gamma oscillations reflect cognitive functions and voluntary motor activities [59]. Coherence of gamma frequency band persists during slow movement, and studies have reported a decrease in the gamma band coherence in patients who did not recover well from stroke [59]. It is thought that lower corticomuscular coherence reflects either a lack in brain-muscle communication, or poorly integrated signals between the brain and muscles during motor actions [59].

We suggest that the brain, astrocytes, neurons, interneurons, heart, and periphery (sensory organs and limbs) pulsate at nearly the same frequency as the brain, which in turn, allows a person to respond and react to the external world in real time. For example, it is known that beta band oscillations in the somatosensory cortex are synchronized with the motor cortex oscillations, which allow oscillatory sensory reafference to correctly interpret oscillatory motor commands [62], and the motor cortex oscillates in the beta band frequency of about $20 \mathrm{~Hz}$ with the muscles contracting contralateral side of the body [62].

Additionally, it has been shown that subthreshold oscillations are evident in membrane potentials of primary sensory neurons in rat dorsal root ganglia cells [63]. These oscillations were voltage sensitive in amplitude, frequency, and coherence, and it was discovered that upon reaching threshold, oscillations gave rise to action potentials [63]. Furthermore, oscillations influence biochemistry and could play a role in diabetes mellitus [64], and aberrant oscillatory patterns have been shown to be concurrent with severe disease and defects (i.e. primary ciliary dyskinesia), or play a significant role in the development of other disorders due to disruption of cellular oscillations that regulate the circadian clock (i.e. 
metabolic syndrome and cognitive disorders related to schizophrenia) [16]. These studies suggest that oscillations play a significant role in internal functions of the body, which affects the consciousness experience.

\section{Conclusion}

This article presents evidence from studies on oscillations, respiration, and membrane potentials to support a theory that consciousness is body-wide biological process not limited to individual organs. Current research regarding the location where consciousness emerges is at best, a fragmented approach. We contend that the mind and body are unified and work together as one entity, and suggest that consciousness exists throughout the entire body. This theory promotes consciousness as a unified experience that is maintained through respiration and membrane potentials because respiration is essential in the body's ability to continue to function. Without respiration, consciousness is terminated. Furthermore, in order for a person to have a complete and fully-developed consciousness experience, the brain, entire body, respiration, cellular and molecular changes, and membrane potentials must all work in unison. More research is needed to connect consciousness to oscillations, respiration, and membrane potentials, to further explore this theory.

\section{References}

[1] Critchley, H.D., Eccles, J. and Garfinkel, S.N. (2013) Interaction between Cognition, Emotion, and the Autonomic Nervous System. Handbook of Clinical Neurology, 117, 59-77. https://doi.org/10.1016/B978-0-444-53491-0.00006-7

[2] Vickery, O.N., Machtens, J.P. and Zachariae, U. (2016) Membrane Potentials Regulating GPCRs: Insights from Experiments and Molecular Dynamics Simulations. Current Opinion in Pharmacology, 30, 44-50. https://doi.org/10.1016/j.coph.2016.06.011

[3] Jerath, R., et al. (2015) Widespread Depolarization during Expiration: A Source of Respiratory Drive? Medical Hypotheses, 84, 31-37. https://doi.org/10.1016/j.mehy.2014.11.010

[4] Sedlmeier, P. and Srinivas, K. (2016) How Do Theories of Cognition and Consciousness in Ancient Indian Thought Systems Relate to Current Western Theorizing and Research? Frontiers in Psychology, 7, 343. https://doi.org/10.3389/fpsyg.2016.00343

[5] Jerath, R., Barnes, V.A. and Crawford, M.W. (2014) Mind-Body Response and Neurophysiological Changes during Stress and Meditation: Central Role of Homeostasis. Journal of BIOLOGICAL REGULATORS \& Homeostatic Agents, 28, 545-554.

[6] Jerath, R., Crawford, M.W. and Barnes, V.A. (2015) A Unified 3D Default Space Consciousness Model Combining Neurological and Physiological Processes That Underlie Conscious Experience. Frontiers in Psychology, 6, 1204. https://doi.org/10.3389/fpsyg.2015.01204

[7] Guyton, A.C. and Hall, J.E. (2000) Textbook of Medical Physiology. 10th Edition, W.B. Saunders, Philadelphia.

[8] Klabunde, R. (2011) Cardiovascular Physiology Concepts. Lippincott Williams \& Wilkins, Philadelphia. 
[9] Veech, R.L., Kashiwaya, Y. and King, M.T. (1995) The Resting Membrane Potential of Cells Are Measures of Electrical Work, Not of Ionic Currents. Integrative Psychological and Behavioral Science, 30, 283-307. https://doi.org/10.1007/BF02691602

[10] Carrasquillo, Y., Burkhalter, A. and Nerbonne, J.M. (20120 A-Type K+ Channels Encoded by Kv4.2, Kv4.3 and Kv1.4 Differentially Regulate Intrinsic Excitability of Cortical Pyramidal Neurons. Journal of Physiology, 590, 3877-3890.

https://doi.org/10.1113/jphysiol.2012.229013

[11] Fischer, H. (2012) Function of Proton Channels in Lung Epithelia. Wiley Interdisciplinary Reviews: Membrane Transport and Signaling, 1, 247-258. https://doi.org/10.1002/wmts.17

[12] Cheng, K., Haspel, H.C., Vallano, M., Osotimehin, B. and Sonenberg, M. (1980) Measurement of Membrane Potentials (Psi) of Erythrocytes and White Adipocytes by the Accumulation of Triphenylmethylphosphonium Cation. Journal of Membrane Biology, 56, 191-201. https://doi.org/10.1007/BF01869476

[13] Jerath, R. and Crawford, M.W. (2015) Layers of Human Brain Activity: A Functional Model Based on the Default Mode Network and Slow Oscillations. Frontiers in Human Neuroscience, 9, 248. https://doi.org/10.3389/fnhum.2015.00248

[14] Kay, L.M., et al. (2009) Olfactory Oscillations: The What, How and What for. Trends in Neurosciences, 32, 207-214. https://doi.org/10.1016/j.tins.2008.11.008

[15] Zelano, C., et al. (2016) Nasal Respiration Entrains Human Limbic Oscillations and Modulates Cognitive Function. Journal of Neuroscience, 36, 12448-12467. https://doi.org/10.1523/JNEUROSCI.2586-16.2016

[16] Muehsam, D. and Ventura, C. (2014) Life Rhythm as a Symphony of Oscillatory Patterns: Electromagnetic Energy and Sound Vibration Modulates Gene Expression for Biological Signaling and Healing. Global Advances in Health and Medicine, 3, 40-55. https://doi.org/10.7453/gahmj.2014.008

[17] Jerath, R., Crawford, M.W. and Barnes, V.A. (2015) Functional Representation of Vision within the Mind: A Visual Consciousness Model Based in 3D Default Space. Journal of Medical Hypotheses and Ideas, 9, 45-56. https://doi.org/10.1016/j.jmhi.2015.02.001

[18] Jerath, R. and Crawford, M.W. (2015) How Does the Body Affect the Mind? Role of Cardiorespiratory Coherence in Spectrum of Emotions. Advances in Mind-Body Medicine, 29, 4-16.

[19] Jerath, R., Cearley, S., Barnes, V. and Nixon-Shapiro, E. (2016) How Lateral Inhibition and Fast Retinogeniculo-Cortical Oscillations Create Vision: A New Hypothesis. Medical Hypotheses, 96, 20-29. https://doi.org/10.1016/j.mehy.2016.09.015

[20] Sherman, S.M. (2005) Thalamic Relays and Cortical Functioning. Progress in Brain Research, 149, 107-126. https://doi.org/10.1016/S0079-6123(05)49009-3

[21] Saalmann, Y.B. and Kastner, S. (2011) Cognitive and Perceptual Functions of the Visual Thalamus. Neuron, 71, 209-223. https://doi.org/10.1016/j.neuron.2011.06.027

[22] Fuentealba, P., Timofeev, I., Bazhenov, M., Sejnowski, T.J. and Steriade, M. (2005) Membrane Bistability in Thalamic Reticular Neurons during Spindle Oscillations. Journal of Neurophysiology, 93, 294-304. https://doi.org/10.1152/jn.00552.2004

[23] Wang, X., Sommer, F.T. and Hirsch, J.A. (2011) Inhibitory Circuits for Visual Processing in Thalamus. Current Opinion in Neurobiology, 21, 726-733. https://doi.org/10.1016/j.conb.2011.06.004

[24] Niculescu, D. and Lohmann, C. (2014) Gap Junctions in Developing Thalamic and Neocortical Neuronal Networks. Cerebral Cortex, 24, 3097-3106. 
https://doi.org/10.1093/cercor/bht175

[25] Steriade, M. and Timofeev, I. (2003) Neuronal Plasticity in Thalamocortical Networks during Sleep and Waking Oscillations. Neuron, 37, 563-576. https://doi.org/10.1016/S0896-6273(03)00065-5

[26] Liu, K.K., Bartsch, R., Lin, A., Mantegna, R. and Ivanov, P. (2015) Plasticity of Brain Wave Network Interactions and Evolution across Physiologic States. Frontiers in Neural Circuits, 9, 62. https://doi.org/10.3389/fncir.2015.00062

[27] Sejnowski, T.J. and Destexhe, A. (2000) Why Do We Sleep? Brain Research, 886, 208-223. https://doi.org/10.1016/S0006-8993(00)03007-9

[28] Purves, D., Augustine, G.J., Fitzpatrick, D., Katz, L., LaMantia, A.-S., McNamara, J. and Williams, S.M. (2001) Thalamocortical Interactions. In: Purves, D., et al., Eds,, Neuroscience, 2nd Edition, Sinauer Associates, Sunderland, 618.

[29] Montecino-Rodriguez, E. and Dorshkind, K. (2001) Regulation of Hematopoiesis by Gap Junction-Mediated Intercellular Communication. Journal of Leukocyte Biology, 70, 341-347.

[30] Gonzalez-Nieto, D., et al. (2015) Connexins: Intercellular Signal Transmitters in Lymphohematopoietic Tissues. International Review of Cell and Molecular Biology, 318, 27-62. https://doi.org/10.1016/bs.ircmb.2015.06.001

[31] Retamal, M.A., et al. (2015) Diseases Associated with Leaky Hemichannels. Frontiers in Cellular Neuroscience, 9, 267. https://doi.org/10.3389/fncel.2015.00267

[32] Thorpe, S., Fize, D. and Marlot, C. (1996) Speed of Processing in the Human Visual System. Nature, 381, 520-522. https://doi.org/10.1038/381520a0

[33] Laskowski, M., et al. (2016) What Do We Not Know about Mitochondrial Potassium Channels? Biochimica et Biophysica Acta, 1857, 1247-1257.

https://doi.org/10.1016/j.bbabio.2016.03.007

[34] Marreiros, B.C., et al. (2016) Exploring Membrane Respiratory Chains. Biochimica et Biophysica Acta, 1857, 1039-1067. https://doi.org/10.1016/j.bbabio.2016.03.028

[35] Pittman, R.N. (2011) The Circulatory System and Oxygen Transport.

[36] Homma, I. and Masaoka, Y. (2008) Breathing Rhythms and Emotions. Experimental Physiology, 93, 1011-1021. https://doi.org/10.1113/expphysiol.2008.042424

[37] Jerath, R. (2010) Pranayama: Converting Stress \& Anxiety into Inner Joy. Author House, Bloomington, 103.

[38] Ramirez, J.M., et al. (2012) The Cellular Building Blocks of Breathing. Comprehensive Physiology, 2, 2683-2731. https://doi.org/10.1002/cphy.c110033

[39] Jerath, R. and Barnes, V.A. (2009) Augmentation of Mind-Body Therapy and Role of Deep Slow Breathing. Journal of Complementary and Integrative Medicine, 6, Article Number: 31. https://doi.org/10.2202/1553-3840.1299

[40] Jerath, R., Crawford, M.W., Barnes, V.A. and Harden, K. (2015) Self-Regulation of Breathing as a Primary Treatment for Anxiety. Applied Psychophysiology and Biofeedback, 40, 107-115. https://doi.org/10.1007/s10484-015-9279-8

[41] Jerath, R., Harden, K., Crawford, M., Barnes, V. and Jensen, M. (2014) Role of Cardiorespiratory Synchronization and Sleep Physiology: Effects on Membrane Potential in the Restorative Functions of Sleep. Sleep Medicine, 15, 279-288. https://doi.org/10.1016/j.sleep.2013.10.017

[42] Jerath, R., Edry, J.W., Barnes, V.A. and Jerath, V. (2006) Physiology of Long Pranayamic Breathing: Neural Respiratory Elements May Provide a Mechanism That Explains How Slow Deep Breathing Shifts the Autonomic Nervous System. Medical Hypotheses, 67, 566-571. https://doi.org/10.1016/j.mehy.2006.02.042 
[43] Magnin, M., et al. (2010) Thalamic Deactivation at Sleep Onset Precedes That of the Cerebral Cortex in Humans. Proceedings of the National Academy of Sciences of the United States of America, 107, 3829-3833. https://doi.org/10.1073/pnas.0909710107

[44] Vaschillo, E.G., et al. (2015) The Effects of Sighing on the Cardiovascular System. Biological Psychology, 106, 86-95. https://doi.org/10.1016/j.biopsycho.2015.02.007

[45] Ramirez, J.M. (2014) The Integrative Role of the Sigh in Psychology, Physiology, Pathology, and Neurobiology. Progress in Brain Research, 209, 91-129. https://doi.org/10.1016/B978-0-444-63274-6.00006-0

[46] Choi, S., et al. (2010) Subthreshold Membrane Potential Oscillations in Inferior Olive Neurons Are Dynamically Regulated by P/Q- and T-Type Calcium Channels: A Study in Mutant Mice. Journal of Physiology, 588, 3031-3043.

https://doi.org/10.1113/jphysiol.2009.184705

[47] Cipolla, M.J. (2009) Control of Cerebral Blood Flow.

[48] Buzsaki, G., Kaila, K. and Raichle, M. (2007) Inhibition and Brain Work. Neuron, 56, 771-783. https://doi.org/10.1016/j.neuron.2007.11.008

[49] Vollmer, L.L., Strawn, J.R. and Sah, R. (2015) Acid-Base Dysregulation and Chemosensory Mechanisms in Panic Disorder: A Translational Update. Translational Psychiatry, 5, e572. https://doi.org/10.1038/tp.2015.67

[50] Ayers, P., Dixon, C. and Mays, A. (2015) Acid-Base Disorders Learning the Basics. Nutrition in Clinical Practice, 30, 14-20. https://doi.org/10.1177/0884533614562842

[51] Guyenet, P.G. (2014) Regulation of Breathing and Autonomic Outflows by Chemoreceptors. Comprehensive Physiology, 4, 1511-1562.

https://doi.org/10.1002/cphy.c140004

[52] Shell, B., Faulk, K. and Cunningham, J.T. (2016) Neural Control of Blood Pressure in Chronic Intermittent Hypoxia. Current Hypertension Reports, 18, 19. https://doi.org/10.1007/s11906-016-0627-8

[53] Perry-Parrish, C., et al. (2016) Improving Self-Regulation in Adolescents: Current Evidence for the Role of Mindfulness-Based Cognitive Therapy. Adolescent Health, Medicine and Therapeutics, 7, 101-108. https://doi.org/10.2147/AHMT.S65820

[54] Rosenzweig, S., et al. (2007) Mindfulness-Based Stress Reduction Is Associated with Improved Glycemic Control in Type 2 Diabetes Mellitus: A Pilot Study. Alternative Therapies in Health and Medicine, 13, 36-38.

[55] Esch, T., Fricchione, G.L. and Stefano, G.B. (2003) The Therapeutic Use of the Relaxation Response in Stress-Related Diseases. Medical Science Monitor, 9, RA23RA34.

[56] $\mathrm{Wu}$, S.D. and Lo, P.C. (2010) Cardiorespiratory Phase Synchronization during Normal Rest and Inward-Attention Meditation. International Journal of Cardiolo$g y, 141,325-328$. https://doi.org/10.1016/j.ijcard.2008.11.137

[57] Hoffman, J.W., et al. (1982) Reduced Sympathetic Nervous System Responsivity Associated with the Relaxation Response. Science, 215, 190-192. https://doi.org/10.1126/science.7031901

[58] Salenius, S. and Hari, R. (2003) Synchronous Cortical Oscillatory Activity during Motor Action. Current Opinion in Neurobiology, 13, 678-684. https://doi.org/10.1016/j.conb.2003.10.008

[59] Fang, Y., et al. (2009) Functional Corticomuscular Connection during Reaching Is Weakened Following Stroke. Clinical Neurophysiology, 120, 994-1002. https://doi.org/10.1016/j.clinph.2009.02.173

[60] Mima, T. and Hallett, M. (1999) Corticomuscular Coherence: A Review. Journal of 
Clinical Neurophysiology, 16, 501-511.

https://doi.org/10.1097/00004691-199911000-00002

[61] Wach, C., et al. (2013) The Effect of $10 \mathrm{~Hz}$ Transcranial Alternating Current Stimulation (tACS) on Corticomuscular Coherence. Frontiers in Human Neuroscience, 7, 511. https://doi.org/10.3389/fnhum.2013.00511

[62] Baker, S.N. (2007) Oscillatory Interactions between Sensorimotor Cortex and the Periphery. Current Opinion in Neurobiology, 17, 649-655. https://doi.org/10.1016/j.conb.2008.01.007

[63] Amir, R., Michaelis, M. and Devor, M. (1999) Membrane Potential Oscillations in Dorsal Root Ganglion Neurons: Role in Normal Electrogenesis and Neuropathic Pain. Journal of Neuroscience, 19, 8589-8596.

[64] Ainscow, E.K. and Rutter, G.A. (2001) Mitochondrial Priming Modifies $\mathrm{Ca}^{2+}$ Oscillations and Insulin Secretion in Pancreatic Islets. Biochemical Journal, 353, 175180. https://doi.org/10.1042/bj3530175

Submit or recommend next manuscript to SCIRP and we will provide best service for you:

Accepting pre-submission inquiries through Email, Facebook, LinkedIn, Twitter, etc. A wide selection of journals (inclusive of 9 subjects, more than 200 journals)

Providing 24-hour high-quality service

User-friendly online submission system

Fair and swift peer-review system

Efficient typesetting and proofreading procedure

Display of the result of downloads and visits, as well as the number of cited articles

Maximum dissemination of your research work

Submit your manuscript at: http://papersubmission.scirp.org/

Or contactwjins@scirp.org 